Rev. salud pública. 11 (6): 865-877, 2009

\title{
Prevalência de doenças crônicas e estado nutricional em um grupo de idosos brasileiros
}

\author{
The prevalence of chronic disease in a group of elderly Brazilian \\ people and their nutritional status
}

Christiane Leite-Cavalcanti ${ }^{1}$, Maria da Conceição Rodrigues-Gonçalves ${ }^{1}$, Luiza Sonia Rios-Asciutti ${ }^{1}$ e Alessandro Leite-Cavalcanti ${ }^{2}$

1 Universidade Federal da Paraíba, João Pessoa, Paraíba, Brasil. chris_tiane2006@hotmail.com; raulceica@ig.com.br; luiza.asciutti@terra.com.br.

2 Universidade Estadual da Paraíba, Campina Grande, Paraíba, Brasil. dralessandro@ibest.com.br

Recebido em 4 Maio 2009/Enviado para Modificação em 7 Novembro 2009/Aprovado em 15 Novembro 2009

\section{RESUMO}

Objetivo Avaliar a prevalência de doenças crônicas e o estado nutricional de um grupo de idosos do município de João Pessoa, PB, Brasil.

Método Foram avaliados 117 idosos com idades entre 60 e 89 anos atendidos nos Centros de Referência e Cidadania. $O$ instrumento de pesquisa compreendeu um questionário e incluía variáveis sócio-demográficas, sócio-econômicas, comportamentos relacionados à saúde e antropométricas. Os resultados foram organizados com o Software SPSS, sendo apresentados por meio da estatística descritiva (média e desvio-padrão, freqüências absoluta e percentual).

Resultados Em relação ao uso do tabaco e do álcool, 4,3 \% e 9,4 \%, respectivamente, afirmaram fazer uso destas substâncias e 56,4 \% reportaram não praticar atividade física. Da amostra, 78,6 \% utilizam medicamentos e 82,1\% afirmaram possuir alguma doença crônica não-transmissível, sendo mais freqüentes a hipertensão arterial $(56,4 \%)$, as dislipidemias $(33,3 \%)$ e o diabetes mellitus $(20,5 \%)$. Segundo a classificação do IMC, 46,2 \% apresentavam sobrepeso e 40,2 \% obesidade grau, enquanto em relação ao RCQ, 97,4 \% foram classificados como obesos abdominais. Conclusão A prevalência de doenças crônicas foi elevada, principalmente a hipertensão arterial, com a maioria dos idosos sendo classificados como obesos abdominais, com risco muito alto para desenvolvimento de doenças cardiovasculares.

Palavras-Chave: Avaliação nutricional, Saúde do idoso institucionalizado, Sobrepeso, Obesidade (fonte: DeCS, BIREME).

\section{ABSTRACT}

Objective Evaluating the prevalence of chronic disease in a group of elderly people living in the city of João Pessoa, PB, Brazil and their nutritional status.

Method 117 subjects aged 60 to 89 attending local Reference and Citizenship Centres were evaluated. The research instrument used was a questionnaire addressing 
socio-demographic, socioeconomic and anthropometric variables and health-related behaviour. The data so collected was organised using SPSS statistical software and presented via descriptive statistics (mean and standard deviation, and absolute and percentage frequencies).

Results $4.3 \%$ and $9.4 \%$ of the subjects admitted using tobacco and alcohol, respectively. $56.4 \%$ did not engage in any physical activity. As many as $78.6 \%$ used medications and $82.1 \%$ admitted to suffering from some non-transmissible chronic disease, the most frequent being hypertension (56.4 \%), dyslipidaemia (33.3 \%) and diabetes mellitus $(20.5 \%)$. According to body mass index (BMI) classification, $46.2 \%$ were overweight and $40.2 \%$ presented degree I obesity. Regarding the waist-to-hip ratio (WHR), abdominal obesity was diagnosed in $97.4 \%$ of the elderly in the study.

Conclusion The prevalence of chronic disease, especially hypertension, was high in the studied population. Most institutionalised elderly were classified as suffering from abdominal obesity, representing an extremely high risk of developing cardiovascular disease.

Key Words: Nutritional assessment, health services for the institutionalised elderly, overweight, obesity (source: MeSH, NLM).

\section{RESUMEN}

Prevalencia de enfermedades crónicas y estado nutricional en un grupo de personas de edad avanzada, de Brasil

Objetivo Evaluar la prevalencia de enfermedades crónicas y el estado nutricional de grupo de personas de edad avanzad, del muncipio João Pessoa, PB, Brasil.

Método Se evaluaron 117 personas con edades entre 60 y 89 años, atendidos en los Centros de Referencia y Ciudadanía. Se utilizó un cuestionario que incluía variables socio-demográficas, socio-económicas, comportamientos relacionados con las salud y antropométricas. Los resultados fueron organizados con el software SPSS y se presentaron por medio de estadísticas descriptivas (frecuencias absolutas y proporcionales, medias y desviaciones).

Resultados En relación con el consumo de tabaco y del alcohol, 4,3 y 9,4\% respectivamente afirmaron utilizar estas sustancias y 56,4 \% reportaron no hacer actividad física. Del total, 78,6 \% utilizan medicamentos y $82,1 \%$ afirmaron tener alguna enfermedad crónica no transmisible, más frecuentes la hipertensión arterial $(56,4 \%)$, las dislipidemias $(33,3 \%)$ y la Diabetes mellitus $(20,5$ $\%)$. Con la clasificación del IMC, $46,2 \%$ presentaron sobrepeso y $40,2 \%$ obesidad, mientras que en relación con RCQ, 97,4 \% fueron clasificados como obesos abdominales.

Conclusiones La prevalencia de enfermedades crónicas fue elevada, principalmente la hipertensión arterial, con la mayoría de quienes la presentaron clasificados como obesos abdominales, con alto riesgo de desarrollo de enfermedades cardiovasculares.

Palabras Clave: Evaluación nutricional, salud del anciano institucionalizado, sobrepeso, obesidad (fuente: DeCS, BIREME). 
$\mathrm{O}$ envelhecimento da população é um fenômeno de amplitude mundial, posto que a World Health Organization (WHO) prevê que em 2025 existirão 1,2 bilhões de pessoas com mais de 60 anos, sendo que os muito idosos (com 80 ou mais anos) constituem o grupo etário de maior crescimento (1).

Todavia, esse processo ganha maior importância nos países em desenvolvimento, com o crescimento acelerado da população de sessenta anos e mais em relação à população geral. Aumentos de até $300 \%$ da população idosa são esperados nesses países, especialmente nos que integram a América Latina (2). No Brasil, o aumento da população idosa vem ocorrendo de forma muito rápida, sem a correspondente modificação nas condições de vida (3).

São considerados idosos, nos países em desenvolvimento, os indivíduos com faixa etária igual ou superior a 60 anos de idade enquanto que nos países desenvolvidos o recorte etário é de 65 anos. Em relação ao Brasil, a Lei de ${ }^{\circ}$ 8.842/94, em seu artigo $2^{\circ}$, parágrafo único, refere que "são consideradas idosas as pessoas maiores de 60 anos, de ambos os gêneros, sem distinção de cor, etnia e ideologia" (4).

Vários são os meios de melhorar a qualidade de vida daqueles que estão no processo de envelhecer, dentre os quais se inclui o desenvolvimento de programas de saúde. Esses programas de saúde, que têm como base à comunidade e que implicam promoção da saúde do idoso, devem ter a nutrição como uma das áreas prioritárias (3).

A condição de nutrição é aspecto importante nesse contexto, visto que os idosos apresentam condições peculiares que comprometem seu estado nutricional. Alguns desses condicionantes ocorrem devido às alterações fisiológicas do próprio envelhecimento, enquanto outros são acarretados pelas enfermidades presentes, pelas práticas ao longo da vida (fumo, dieta, atividade física) e situação socioeconômica $(5,6)$.

A manutenção de um estado nutricional adequado é muito importante, pois, de um lado, encontra-se o baixo-peso, que aumenta o risco de infecções e mortalidade, e do outro o sobrepeso, que aumenta o risco de Doenças Crônicas NãoTransmissíveis (DCNT), como hipertensão, diabetes mellitus e hiperlipidemias (7).

Com o fenômeno de envelhecimento populacional, aumenta, cada vez mais, a necessidade de conhecimento dos fatores que incidem sobre a prevalência das DCNT associadas à idade. Um exemplo disso é que taxas elevadas de sobrepeso 
e obesidade em todas as faixas etárias, incluindo os idosos, atingindo os dois gêneros, estão sendo observadas no mundo inteiro (8).

O processo de envelhecimento populacional vem-se constituindo num dos maiores desafios para a saúde pública contemporânea, principalmente nos países onde esse fenômeno tem ocorrido em situações de pobreza e grande desigualdade social (9). O delineamento de políticas específicas para pessoas idosas vem sendo apontado como altamente necessário, sendo imprescindível o conhecimento das necessidades e condições de vida desse grupo etário (10).

Com o aumento de pessoas acima dos 60 anos de idade, eleva-se a necessidade de estudos que investiguem os comportamentos relacionados à saúde e o estado nutricional para que as propostas de políticas de saúde causem impacto na qualidade de vida desta população. Portanto, objetivando oferecer subsídios para o planejamento local de ações de saúde, o presente estudo avaliou a prevalência de doenças crônicas e o estado nutricional em um grupo de idosos do município de João Pessoa, PB, Brasil.

\section{METODOLOGIA}

A pesquisa foi realizada em João Pessoa, capital do Estado da Paraíba, localizada na região nordeste do Brasil. O município possui uma população de mais de 700 mil habitantes e um Índice de Desenvolvimento Humano (IDH) de 0,78.

Local do estudo

Esse estudo epidemiológico e transversal foi realizado nos Centros de Referência e Cidadania (CRC), pertencentes à Secretaria do Desenvolvimento Social (SEDES), da Prefeitura do Município de João Pessoa, PB. Esses centros constituem-se em espaços privilegiados voltados à valorização dos idosos, com o desenvolvimento de atividades de lazer, trabalhos manuais, noções de saúde e o exercício da cidadania. Existem dez centros, distribuídos em diferentes bairros do Município, dos quais nove foram incluídos na pesquisa.

População estudada

De um total de 224 idosos de ambos os gêneros, com idade entre 60 a 87 anos, 117 $(52,2 \%)$ concordaram em participar da pesquisa, sendo a amostra do tipo não probabilística.

Foram excluídos do estudo os idosos que apresentavam alterações de ordem neurológica que os impossibilitassem de compreender os objetivos do estudo e/ou alterações físicas que impossibilitassema verificação das medidas antropométricas. 
Esta pesquisa foi norteada segundo a Resolução 196/96 do Conselho Nacional de Saúde do Brasil e segue os princípios da Declaração de Helsinque, sendo aprovada pelo Comitê de Ética em Pesquisa do Centro de Ciências da Saúde da Universidade Federal da Paraíba - CEP/CCS, sob o nº 680/06.

Instrumento de Pesquisa e Variáveis

Para a caracterização dos idosos, o instrumento de pesquisa compreendeu um questionário individual e incluíam as seguintes variáveis: características sóciodemográficas (gênero, idade e estado civil), comportamentos relacionados à saúde (ingestão de bebida alcoólica, hábito de tabagismo, prática de atividade física, uso de medicamentos, presença e número de doenças crônicas) e antropométricas (peso, estatura, circunferência da cintura e do quadril).

\section{Avaliação Antropométrica}

A coleta de dados foi realizada no período de março a maio de 2007, nos próprios centros onde os idosos eram cadastrados, por um único pesquisador. A avaliação antropométrica foi feita a partir da obtenção das medidas de peso, altura e circunferências de cintura e de quadril (11).

O peso foi verificado utilizando-se uma balança digital (Tech Line TEC-130, capacidade para $136 \mathrm{~kg}$ e intervalo de $100 \mathrm{~g}$ ), com o indivíduo descalço e, com roupas leves. A estatura foi verificada usando-se estadiômetro (Sanny ES 2040, tipo trena com $220 \mathrm{~cm}$ ) com o indivíduo ereto e com os calcanhares alinhados. Foi utilizado o Índice de Massa Corpórea (IMC) considerando-se a razão peso atual $(\mathrm{kg}) / q u a d r a d o$ da estatura $\left(\mathrm{m}^{2}\right)$, baseando-se nos pontos de corte previamente descritos na literatura (12).

As circunferências da cintura (CC) e do quadril (CQ) foram verificadas com o auxílio de uma Fita de Medidas Antropométricas, (Sanny, com $200 \mathrm{~cm}$, divisão de $1 \mathrm{~mm}$ ). ACC foi medida na cintura natural, ou seja, entre as costelas inferiores e as cristas ilíacas; com a leitura feita no momento da expiração, e realizou-se no milímetro mais próximo. ACQ foi verificada no nível da sínfise púbica com a fita circundando o quadril na parte mais saliente entre a cintura e a coxa. A razão cintura/quadril e a CC foram utilizadas como prognóstico de determinação para risco de doença coronariana e cardiovascular (13). 
Análise Estatística

Os resultados obtidos foram organizados com o auxílio do Software SPSS (Statistical Package for the Social Sciences) versão 10.0. Para a descrição das variáveis contínuas utilizou-se a média aritmética, com seu respectivo desvio-padrão, e para as variáveis categóricas, a frequiência absoluta e percentual.

\section{RESULTADOS}

Em relação à caracterização dos idosos, a maioria compreendia mulheres $(94,0$ $\%)$, com idades entre 60 e 69 anos $(55,5 \%$ ) e casadas (35,9\%), conforme pode ser visto na Tabela 1.

\begin{tabular}{|c|c|c|}
\hline \multirow[b]{2}{*}{ Variavéis } & \multicolumn{2}{|c|}{ Freqüência } \\
\hline & $\mathrm{n}$ & $\%$ \\
\hline \multicolumn{3}{|l|}{ Gênero } \\
\hline Feminino & 110 & 94,0 \\
\hline Masculino & 7 & 6,0 \\
\hline \multicolumn{3}{|c|}{ Faixa Etária (em anos) } \\
\hline 60 a 69 & 65 & 55,6 \\
\hline 70 a 79 & 41 & 35,0 \\
\hline$\geq 80$ & 11 & 9,4 \\
\hline \multicolumn{3}{|l|}{ Estado Civil } \\
\hline Casado & 42 & 35,9 \\
\hline Viúvo & 54 & 46,2 \\
\hline Solteiro & 14 & 12,0 \\
\hline Divorciado & 7 & 6,0 \\
\hline
\end{tabular}

Quando investigados sobre o uso do tabaco, apenas 4,3\% dos idosos informaram possuir este hábito. Em relação ao uso do álcool, 9,4 \% relataram utilizá-lo. A prática regular de atividade física foi descrita por 43,6\% dos idosos (Tabela 2).

Em relação ao estado de saúde, 78,6 \% dos idosos relataram utilizar algum tipo de medicamento e 82,1 \% afirmaram possuir alguma doença crônica nãotransmissível. Dentre aqueles que relataram ser portadores, a maioria $(37,6 \%)$ informou ter uma única patologia crônica não-transmissível. 
Tabela 2. Distribuição dos idosos segundo os hábitos de tabagismo, ingestão de álcool e prática de atividade física João Pessoa/PB, Brasil, 2007

\begin{tabular}{lcc}
\hline Hábitos de vida & $\mathrm{n}$ & Freqüência \\
\hline Tabagismo & 5 & 4,3 \\
Sim & 112 & 95,7 \\
Não & & \\
Ingestão de Álcool & 11 & 9,4 \\
Sim & 106 & 90,6 \\
Não & & \\
Prática de Atividade Física & 51 & 43,6 \\
Sim & 66 & 56,4 \\
Não &
\end{tabular}

Tabela 3. Distribuição dos idosos segundo o uso de medicamentos, presença e número de doenças crônicas não-transmissíveis existentes João Pessoa/PB, Brasil, 2007

\begin{tabular}{lcc}
\hline Estado de Saúde & N & $\%$ \\
\hline Uso de Medicamentos & 92 & 78,6 \\
Sim & 25 & 21,4 \\
Não & & \\
Presença de DCNT & 96 & 82,1 \\
Sim & 21 & 17,9 \\
Não & 44 & 37,6 \\
Número de DCNT & 28 & 23,9 \\
1 & 22 & 18,8 \\
2 & 2 & 1,7 \\
3 & & \\
4 &
\end{tabular}

Em relação ao tipo de doença crônica não-transmissível existente, as mais recorrentes foram a hipertensão arterial $(56,4 \%)$, seguida de dislipidemias $(33,3 \%)$ e Diabetes mellitus (20,5\%), conforme demonstrado na Tabela 4.

Tabela 4. Distribuição dos idosos segundo o tipo de doença crônica não-transmissível existente. João Pessoa/PB, Brasil, 2007

\begin{tabular}{lcc}
\multicolumn{1}{c}{ DCNT } & Freqüência & $\%$ \\
\hline Hipertensão Arterial Sistêmica & 66 & 56,4 \\
\hline Dislipidemias & 39 & 33,3 \\
Diabetes Mellitus & 24 & 20,5 \\
Doença Cardiaca & 23 & 19,6 \\
Osteoporose & 14 & 12 \\
\hline Artrite/Artrose & 5 & 4,3 \\
Acidente Vascular Cerebral $_{\text {Hepatopatias }}$ & 3 & 2,5 \\
Doença Renal $^{1}$ & 2 & 1,7 \\
Total $^{1}$ & 1 & 0,8 \\
(1)Podia ser registrado mais de 1 tipo de doença crönica. & 177 & 100,0 \\
\hline
\end{tabular}


Em relação ao peso e à estatura da amostra, observou-se que o peso médio dos idosos foi de $69,3 \mathrm{~kg}$, enquanto a estatura foi de 1,49 metros. Quanto ao IMC, a média foi de $30,98 \mathrm{~kg} / \mathrm{m}^{2}$, sendo a circunferência de cintura de $99,08 \mathrm{~cm}$ e a circunferência de quadril de $105,69 \mathrm{~cm}$. O RCQ foi de 0,93 (Tabela 5).

Tabela 5. Características antropométricas dos idosos João Pessoa/PB, Brasil, 2007

\begin{tabular}{lcc}
\hline \multicolumn{1}{c}{ Variáveis } & Média & Desvio-Padrão \\
\hline Peso $(\mathrm{kg})$ & 69,3 & 9,26 \\
Estatura $(\mathrm{cm})$ & 1,49 & 0,64 \\
IMC $\left(\mathrm{kg} / \mathrm{m}^{2}\right)$ & 30,98 & 3,32 \\
Circunferência de Cintura $(\mathrm{cm})$ & 99,08 & 8,47 \\
Circunferência de Quadril $(\mathrm{cm})$ & 105,69 & 8,26 \\
RCQ & 0,93 & 0,07 \\
\hline
\end{tabular}

Segundo a classificação do IMC, 46,2 \% dos idosos apresentavam sobrepeso e 40,2 \% obesidade grau I. Em relação ao RCQ, 97,4 \% dos mesmos foram classificados como obesos abdominais, com risco muito alto para desenvolvimento de doenças cardiovasculares, conforme demonstrado na Tabela 6.

Tabela 6. Distribuição dos idosos segundo a classificação do IMC e da RCQ João Pessoa, 2007

\begin{tabular}{ccc}
\hline Variáveis & $\mathrm{n}$ & Frequencia \\
& 54 & $\%$ \\
\hline Classificação do IMC & 47 & 46,1 \\
Sobrepeso & 14 & 40,2 \\
Obesidade Grau I & 2 & 12,0 \\
Obesidade Grau II & & 1,7 \\
Obesidade Grau III & 114 & \\
Classificação da RCQ & 2 & 97,4 \\
Obeso Abdominal & 1 & 1,7 \\
Obeso Glúteo & & 0,9 \\
Adequado & & \\
\hline
\end{tabular}

\section{DISCUSSÃO}

A escolha dos Centros de Referência de Cidadania para a coleta dos dados deveu-se ao fato de que os mesmos se constituem em unidades de atendimento a população, incluindo a população idosa do município de João Pessoa e que estão envolvidos em ações que possibilitam e estimulam a participação popular. São espaços que realizam as atividades dos programas sociais em execução e a mobilização comunitária para o exercício da cidadania ativa. Portanto, os resultados aqui descritos descrevem a prevalência de doenças crônicas e o estado nutricional de um grupo específico de idosos. 
A análise da distribuição dos idosos segundo o sexo revelou que a maior parte era composta por idosas do sexo feminino, semelhante ao verificado por outros pesquisadores no Brasil $(3,14)$ e na Colômbia (15). Outra importante consideração deve-se ao fato de que como o estudo foi realizado em Centros de Referência é possível que esta predominância das mulheres seja devido ao fato de que há uma maior procura das mesmas pelo serviço. $\mathrm{O}$ contingente feminino aumenta de maneira mais expressiva que o masculino, pois as mulheres vivem, em média, oito anos a mais que os homens (16).

Corroborando estudos prévios $(17,18)$, verificou-se predomínio de uma população idosa "jovem" no Brasil, situada na faixa de 60 a 69 anos, aspecto que influencia o seu perfil de saúde, visto que pessoas muito idosas são geralmente mais frágeis e demandam serviços de maior complexidade (19).

Em relação aos hábitos de vida, apenas 4,3 \% dos idosos afirmaram ser fumantes, enquanto que em relação ao consumo de álcool menos de $10 \%$ da amostra relataram a ingestão desta substância. $\mathrm{O}$ tabagismo é a principal causa de mortalidade em todo o mundo (20).

A maioria dos idosos deste estudo relatou não praticar nenhum tipo de atividade física. É cediço que a prática de atividade física regular diminui o risco de desenvolver doenças crônicas não-transmissíveis, além de produzir um bemestar físico e mental (21). Deste modo, as pessoas podem manter-se saudáveis depois dos 70, 80 e 90 anos se tiverem uma alimentação equilibrada, mantiverem uma prática regular de atividade física e não fumarem (22).

O uso de medicamentos foi bastante elevado, com um percentual da amostra utilizando 3 ou mais medicamentos simultaneamente. Os idosos costumam utilizar muito mais medicamentos do que pessoas de outra faixa etária; assim, estão mais propensos a sofrer seus efeitos adversos, incluindo as interações medicamento-alimento $(23,24)$.

Quando questionados sobre a presença de alguma doença crônica nãotransmissível, 82,1 \% afirmaram ter ao menos uma doença crônica, corroborando achados prévios $(25,26)$, sendo que $37,6 \%$ relataram possuir uma única doença crônica não-transmissível. Segundo dados do Instituto Brasileiro de Geografia e Estatística inquéritos populacionais realizados no país demonstram que a maioria dos idosos $(80 \%)$ apresenta pelo menos uma doença crônica, e uma significativa parcela, 33\%, três ou mais agravos (27). 
Os dados deste estudo são sugestivos da influência do excesso de peso como fator de risco para a hipertensão, pois mais de $56 \%$ da amostra possuíam esta condição. Corroborando estes achados a literatura revela associação entre o excesso ponderal e o nível de pressão arterial aumentado $(28,29)$. Portanto, a influência adversa que a obesidade exerce em relação à pressão arterial, ao metabolismo da glicose e lipídeos sanguíneos pode levar ao aparecimento de desordens crônicas nas diversas fases da vida $(6,30,31,32)$. Desta forma os dados encontrados nesse estudo, alertam para esse potencial problema.

Outra variável de fundamental importância para conhecer o perfil nutricional de uma população diz respeito ao IMC. Problemas nutricionais estão associados ao aumento da morbidade e mortalidade e com impacto negativo na qualidade de vida entre idosos. Vários pesquisadores têm sugerido a utilização do IMC em estudos para investigar a relação entre sobrepeso e baixo peso com o risco de mortalidade (33).

Na população estudada, a elevada prevalência de sobrepeso e de obesidade, com valores médios de IMC de $30,98 \pm 3,32 \mathrm{~kg} / \mathrm{m}^{2}$, constitui-se fator de risco para a saúde destes idosos, uma vez que valores elevados de IMC podem estar associados a altas taxas de morbidade e mortalidade e uma pior qualidade de vida (34), estando, portanto, em concordância com achados prévios (7,35). Deste modo, idosos com IMC elevado estão mais propensos a apresentarem uma frequiência maior de doenças crônicas não-transmissíveis (15).

O perfil nutricional dos idosos brasileiros a partir dos dados da Pesquisa Nacional sobre Saúde e Nutrição - PNSN/1989 revelou uma prevalência de sobrepeso, o que vem sendo demonstrado em diferentes estudos onde a desnutrição, o sobrepeso e a obesidade predominam sobre os indivíduos eutróficos (6).

A razão cintura/quadril tem sidousada em estudos populacionais como preditora dos riscos de doenças cardiovasculares (36). Os resultados de RCQ demonstraram-se elevados na quase totalidades dos indivíduos estudados, sendo que os valores médios encontrados foram de $0,97 \pm 0,10 \mathrm{~cm}$ e $0,93 \pm 0,07 \mathrm{~cm}$ para homens e mulheres, respectivamente. Esses dados sugerem uma alta predisposição desta população a desenvolver doenças crônico não-transmissíveis, visto que o acúmulo de gordura na região abdominal apresenta estreitas relações com doenças cardiovasculares (7).

Quanto à CC, os valores médios encontrados para ambos os sexos foram acima do recomendado (13). A obesidade e, particularmente, a localização ab- 
dominal de gordura tem grande impacto sobre as doenças cardiovasculares por associarem-se com grande freqüência a condições tais como dislipidemias, hipertensão arterial, resistência à insulina e diabetes, que favorecem a ocorrência de eventos cardiovasculares, particularmente os coronarianos. Independentemente do sobrepeso, a gordura abdominal é importante fator de risco para essas condições $(37,38,39)$.

Uma questão importante abordada sobre obesidade, e que merece atenção, diz respeito ao direcionamento da informação: excesso de peso é sempre danoso à saúde. A detecção precoce de eventual alteração do estado nutricional de uma população é essencial para o desenvolvimento de trabalhos preventivos ou de intervenção terapêutica, evitando o desenvolvimento de doenças no futuro.

Considerando-se que a estruturação das políticas públicas de saúde deve estar fundamentada no diagnóstico de problemas específicos, espera-se que os resultados do presente estudo possam subsidiar programas de promoção, prevenção e atenção ao idoso. Por meio de esforços conjuntos pode-se empenhar numa luta pela diminuição do impacto da desigualdade social e pelo direito de todos ao acesso a melhores condições de vida e saúde, de modo a se garantir um envelhecimento saudável.

A despeito da importância dos achados uma vez que se constitui na primeira caracterização da população acima de 60 anos, é relevante destacar algumas limitações deste estudo. A principal e mais importante limitação é o fato de a pesquisa ter sido feita em centros de referência, de modo que apenas aqueles idosos que estavam cadastrados foram examinados, não possibilitando extrapolar os resultados para toda a população idosa do município de João Pessoa. Outro aspecto se reporta à seleção dos sujeitos pesquisados que foi uma amostragem do tipo não probabilística. Esse tipo de amostragem oferece boas estimativas sobre os parâmetros da população, mas não permite fazer inferências/ generalizações sobre toda a população.

A partir dos resultados obtidos, sugere-se que estudos semelhantes sejam realizados periodicamente, em diversas cidades brasileiras e também em outros países, objetivando conhecer o estado nutricional da população idosa, para que se possa intervir na prevenção de enfermidades e, consequentemente, na melhoria da qualidade de vida desses indivíduos.

Os dados apresentados conferem à população estudada atenção especial, principalmente para o controle do ganho de peso excessivo como fator de risco 
ou como consequiência relativa às doenças crônicas não transmissíveis que podem acarretar um grande impacto no estado nutricional. Por se tratar de um grupo etário em rápido crescimento, as condições de saúde e nutricionais dos idosos são imprescindíveis para o estabelecimento de ações mais efetivas no controle e/ ou prevenção dos fatores relacionados à saúde na terceira idade que resultam do estilo de vida, principalmente em idosos considerados jovens *

\section{REFERÊNCIAS}

1. Sousa L, Galante H, Figueiredo D. Qualidade de vida e bem-estar dos idosos: um estudo exploratório na população portuguesa. Rev Saúde Pública 2003; 37(3):364-71.

2. Giatti L, Barreto SM. Saúde, trabalho e envelhecimento no Brasil. Cad Saúde Pública 2003; 19(3):759-71.

3. Cervato AM, Derntl AM, Latorre MRDO, Marucci MFN. Educação nutricional para adultos e idosos: uma experiência positiva em Universidade Aberta para a Terceira Idade. Rev Nutrição 2005; 18(1):41-52.

4. Brasil. Ministério da Saúde. Estatuto do Idoso. Brasília: Editora MS; 2003.

5. Sampaio LR. Avaliação nutricional e envelhecimento. Rev Nutr 2004; 17(4):507-14.

6. Amado TCF, Arrura IKG, Ferreira RAR. Aspectos alimentares, nutricionais e de saúde de idosas atendidas no Núcleo de Atenção ao Idoso - NAI, Recife 2005. Arch Latinoam Nutr 2007; 57(4):366-72.

7. Bueno JM, Martino HSD, Fernandes MFS, Costa LS, Silva RR. Avaliação nutricional e prevalência de doenças crônicas não transmissíveis em idosos pertencentes a um programa assistencial. Ciênc Saúde Coletiva 2008; 13(4):1237-46.

8. Acuña $\mathrm{K}$, Cruz T. Avaliação do estado nutricional de adultos e idosos e situação nutricional da população brasileira. Arq Bras Endocrinol Metab 2004; 48(3):345-61.

9. Lima-Costa MF, Barreto SM. Tipos de estudos epidemiológicos: conceitos básicos e aplicações na área do envelhecimento. Epidemiol Saúde 2003; 12(4):189-201.

10. Veras R. Em busca de uma assistência adequada à saúde do idoso: revisão da literatura e aplicação de um instrumento de deteç̧ão precoce e de previsibilidade de agravos. Cad Saúde Pública 2003; 19(3):705-15.

11. World Health Organization (WHO). Physical Status: the use and interpretation of anthropometry. Geneva: World Health Organization;1995.

12. Lipschitz DA. Screening for nutritional status in the elderly. Primary Care 1994; 21(1):55-67.

13. World Health Organization (WHO). Obesity: preventing and managing the global epidemic. Geneva:World Health Organization; 1998.

14. Lebrão ML. O projeto SABE em São Paulo: uma visão panorâmica. In: Lebrão ML, Duarte YAO (Orgs.). SABE: Saúde, Bem-estar e Envelhecimento. O Projeto SABE no Município de São Paulo: uma abordagem inicial. Brasília: Organização Pan-Americana de Saúde; 2003.

15. Ramirez-Velez R, Agredo RA, Jerez AM, Chapal LY. Calidad de vida y condiciones de salud en adultos mayores no institucionalizados en Cali, Colombia. Rev Salud Pública (Bogotá) 2008; 10(4):529-36.

16. Instituto Brasileiro de Geografia e Estatística. 2003 [Internet]. Estimativas populacionais. Disponível em : http://www.ibge.org.br. Acesso em: 15 Out. 2006.

17. Cabrera MAS, Jacob Filho W. Obesidade em idosos: prevalência, distribuição e associação com hábitos e co-morbidades. Arq Bras Endocrinol Metab 2001; 45(5):494-501.

18. Pereira RS, Curioni CC, Veras R. Perfil demográfico da população idosa no Brasil e no Rio de Janeiro em 2002. Textos Sobre Envelhecimento 2003; 6(1):43-59.

19. Tavares EL, Anjos LA. Perfil antropométrico da população idosa brasileira. Resultados da Pesquisa Nacional sobre Saúde e Nutrição. Cad Saúde Pública 1999; 15(4):759-68. 
20. Campo-Arias A. Prevalencia de dependencia de nicotina en algunas poblaciones: una revisión sistemática. Rev Salud Publica (Bogotá) 2006; 8(1):98-107.

21. Robledo-Martinez R. Características socioculturales de la actividad física en tres regiones de Colombia. Rev Salud Publica (Bogotá) 2006; 8(2):13-27.

22. World Health Organization (WHO). Diet, nutrition and the prevention of chronic diseases. Report of the joint WHO/FAO expert consultation. Geneva: World Health Organization; 2003.

23. Passero V, Moreira EAM. Estado nutricional de idosos e sua relação com a qualidade de vida. Rev Bras Nut Clín 2003; 18(1):1-7.

24. Rozenfeld $S$. Prevalência, fatores associados e mau uso de medicamentos entre idosos: uma revisão. Cad Saúde Pública 2003; 19(3):717-24.

25. Ramos LR. Fatores determinantes do envelhecimento saudável em idosos residentes em centro urbano: projeto Epidoso, São Paulo. Cad Saúde Pública 2003; 19(3):793-8.

26. Firmo JOA, Barreto SM, Costa MFL. The Bambuí Health and Aging Study (BHAS): factors associated with the treatment of hypertension in older adults in the community. Cad Saúde Pública 2003; 19(3):817-27.

27. Instituto Brasileiro de Geografia e Estatística [Internet]. Censo Demográfico. Disponível em: http:/ /www.ibge.org.br. Acesso em: 10 Out. 2006.

28. El Ayachi M, Mziwira M, Vincent S, Defoort C, Portugal H, Lairon D, Belahsen R. Lipoprotein profile and prevalence of cardiovascular risk factors in urban Moroccan women. Eur J Clin Nutr 2005; 59(12):1379-86

29. Lepira FB, M'buyamba-Kabangu JR, Kayembe KP, Nseka MN. Correlates of serum lipids and lipoproteins in Congolese patients with arterial hypertension. Cardiovasc J S Afr 2005; 16(5):249-55.

30. Banegas JR, Lopez-Garcia E, Gutierrez-Fisac JL, Guallar-Castillon P, Rodriguez- Artalejo F. A simple estimate of mortality attributable to excess weight in the European Union. Eur J Clin Nutr 2003; 2(57):201-18.

31. Empana JP, Ducimetiere P, Charles MA, Jouven X. Sagittal abdominal diameter and risk of sudden death in asymptomatic middle-aged men: the Paris Prospective Study I. Circulation 2004; 110(18):2781-5.

32. Hope AA, Kumanyika SK, Whitt MC, Shults J. Obesity-related comorbidities in obese African Americans in an outpatient weight loss program. Obes Res 2005; 13(4):772-9.

33. Allison DB, Zhu SK, Plankey M, Faith MS, Heo M. Differential associations of body mass index and adiposity with all-cause mortality among men in the first and second National Health and Nutrition Examination Surveys (NHANES I and NHANES II) follow-up studies. Int J Obesity 2002; 26(3):410-6.

34. Heo M, Allison DB, Faith MS, Zhu S, Fontaine KR. Obesity and health-related quality of life: the mediating effects of joint pain and comorbidities. Obes Res 2003; 11(2):209-16.

35. Santos DM, Sichieri R. Índice de massa corporal e indicadores antropométricos de adiposidade em idosos. Rev Saúde Pública 2005; 39(2):163-8.

36. Cabrera MAS, Gebara OCE, Diament J, Nussbacher A, Rosano G Metabolic syndrome abdominal obesity, and cardiovascular risk in elderly women. Int J Cardiol 2007; 114(2):224-9.

37. Poulter N. Global risk of cardiovascular disease. Heart 2003; 89(1):112-5.

38. Grundy SM. Obesity, metabolic syndrome, and cardiovascular disease. J Clin Endocrinol Metab 2004; 89(6):2595-2600.

39. Lang A, Froelicher ES. Management of overweight and obesity in adults: behavioral intervention for long-term weight loss and maintenance. Eur J Cardiovasc Nurs 2006; 5(4):102-14. 